\title{
Editorial
}

\section{New omic and network paradigms for deep understanding of therapeutic mechanisms for Fangji of traditional Chinese medicine}

\author{
Dayue Darrel DUAN ${ }^{1,2, *}$, Zhong WANG ${ }^{3, *}$, Yong-yan WANG ${ }^{3, *}$ \\ ${ }^{1}$ Center for Phenomics of Traditional Chinese Medicine/the Affiliated Hospital of Traditional Chinese Medicine, Southwest Medical \\ University, Luzhou 646000, China; '² Department of Pharmacology, University of Nevada Reno School of Medicine, Reno, NV 89557, \\ USA; ${ }^{3}$ Institute of Basic Research in Clinical Medicine, China Academy of Chinese Medical Sciences, Beijing 100700, China
}

Acta Pharmacologica Sinica (2018) 39: 903-904; doi: 10.1038/aps.2018.42

Combination therapy with multi-drug regimen as an integrated intervention of several pharmacological compounds that interact with multiple targets, rather than monotherapy using a single compound that targets at a single molecule, is a common strategy for combating complex disease in both Western and traditional Chinese medicine $(\mathrm{TCM})^{[1-3]}$. But each is based on different mechanistic principles. In Western medicine, a multi-drug regimen usually combines several monotherapies targeting on different molecules to optimize pharmacodynamics and/or pharmacokinetics to improve therapeutic efficacy and/or reduce toxicity and adverse reactions ${ }^{[1]}$. In TCM, the main therapeutics is the unique TCM medicinal formula, so called Fangji, which is usually composed of multiple herbs and medical materials with integrated multiple therapeutic objects. One of the major principles of Fangji compositions is Zhenghou based "Jun-Chen-Zuo-Shi" to orchestrate and integrate the multiple therapeutic targets for a specific Zhenghou ${ }^{[4]}$. The ingredients in a Fangji were thus composed according to the quaternity of Jun (monarch), which targets at the major etiological mechanisms, Chen (minister), which targets at the secondary etiological mechanisms, Zuo (assistant or associate), which targets at the associated factors to facilitate the therapeutic effects or reduce the adverse effects, and Shi (guide), which guides and orchestrates the targets ${ }^{[4]}$. Therefore, TCM Fangji combination therapy has embodied many principles of modern systems biology and omic theories ${ }^{[2,3]}$. The therapeutic effects of TCM Fangji rely on the integrated whole function through compatibility of the drugs in the multi-drug regimen of Fangij ${ }^{[2]}$. For example, the Duhuo Jisheng Decoc-

\footnotetext{
${ }^{*}$ To whom correspondence should be addressed.

E-mail dduan@medicine.nevada.edu (Dayue Darrel DUAN); zhonw@sina.com (Zhong WANG);

wangyongyan2010@sina.cn (Yong-yan Wang)
}

tion (DHJSD) widely used in clinic for effectively combatting lower back pain (LBP) was developed with an integrated combination of 15-ingredients according to the principles of "Jun, Chen, Zuo, and Shi" to specifically target at the primary and secondary causatives of Bi Zheng ${ }^{[5]}$. It eliminates "feng-hanshi" and thus pain of "Bi Zheng" and promotes "Gan" and "Shen" function and therefore strengthens the bones and tendons, respectively. It also improves circulation and removes stasis of "Qi" and "Xue", reduces tissue swelling and relieves pain. Liu et al found that DHJSD inhibited the generation of proinflammatory factors and extracellular matrix (ECM) degradation of human intervertebral disc (HID) through an orchestrated targeting at multiple molecules in the SDF-1/ CXCR4/NF-kB pathway, thus solved a puzzle of the quartanity of Jun-Chen-Zuo-Shi and added novel mechanistic insights into the clinical effectiveness of DHJSD on $\mathrm{LBP}^{[5]}$. Luo et al studied the Fangji of Yangxin Tongmai formula (YTF) in the treatment of pediatric Graves' disease (GD). They found that YTF enhanced glucose handling in GD children with impaired glucose tolerance through multiple targets to promote the bioactivity of insulin and to avoid the low density of insulin receptors that is induced by the feeble binding affinity of insu$\operatorname{lin}^{[6]}$.

The modern pharmacological approach to the study of Fangji, however, has been focusing on the isolation and identification of individual active components within a Fangji for cellular and molecular targets. Although this approach has led to the development of many new monomers purified from Fangji as new drugs widely used in clinical practice such as the antimalarial artemisinin ${ }^{[7]}$, which has earned a Nobel Prize in Physiology or Medicine in $2015^{[8]}$, the pharmacological bases of these purified effective monomers or active components have lost the TCM characteristics and are far different from the pharmacological theory and clinical applications of Fangji, 
in terms of the principles of combination therapy based on the composition theories such as the quaternity of Jun-Chen-ZuoShi. This not only has led to the misinterpretations of mechanisms of Fangji's therapeutic actions and clinical effectiveness but also has seriously hampered the scientific research and development of TCM in general.

Clearly, new omic/systematic and networking paradigms are urgently needed for deep understanding of the unique composition theories and mechanisms of effective combination therapy through TCM Fangji. Recent endeavors attributed to this revolutionary paradigm shift has resulted in the current special issue of Fangjiomics. Original studies and reviews on the application of genomics ${ }^{[9]}$, transcriptomics $^{[10]}$, proteomics $^{[10]}$, metabolomics ${ }^{[11]}$, fangijomics ${ }^{[12,13]}$ and network pharmacology ${ }^{[12,14]}$ and network medicine ${ }^{[15-19]}$ to the study of several classic Fangjis are incleded. The unbiased genomewide association study (GWAS) and pharmacogenomics have been applied to dissect the genetic variants underlying complex diseases and individual responses to a given treatment ${ }^{[9]}$. Recent development of a full genetic model for analysis of gene-gene interactions (dominance and epistasis) and geneenvironment interactions has substantially increased model power and remarkably improved the detection of association of GWAS and the construction of the molecular architecture $^{[9]}$. This analysis can integrate other omic information and allow for variations of Fangji, which is very promising for Fangiiomic detection of the sophisticated molecular and structural architecture of the function of Fang $\mathrm{j}^{[9]}$.

Zhang et al investigated the mechanisms of Guanxinjing capsules (GXJCs) on coronary heart disease (CHD) complicated with depression ${ }^{[14]}$. A total of 16 GXJC drug-like chemical constituents were identified by UHPLC-LTQ-Orbitrap assay and evaluation of oral bioavailability. Then, 870 genes were identified as the putative targets of these GXJC drug-like chemical constituents by using MedChem Studio. A CHD/depression therapeutic target network of GXJC was then constructed, and four topological features (degree, betweenness, closeness and K-coreness) were calculated. Based on the topological feature values of the GXJC putative targets, 14 main active constituents were identified. Their corresponding putative targets had topological importance in the GXJC putative target-known $\mathrm{CHD} /$ depression therapeutic target network, which were defined as the candidate targets of GXJC against CHD complicated with depression ${ }^{[14]}$. Functionally, these candidate targets were significantly involved in several CHD/depressionrelated pathways, including repairing pathological vascular changes, reducing platelet aggregation and inflammation, and affecting patient depression. Using this integrative pharmacology approach of active chemical constituent identification, drug target prediction and network analysis Zhang et al identified a list of main active constituents of GXJC acting on CHD complicated with depression. This approach to the identification and characterization of molecular targets of Fangji is more efficient and adequate than the conventional pharmacological approach and provides more accurate information for better understanding of the pharmacological mechanisms of TCM prescription of Fangji.

Fangiomics, which was introduced in the previous special issue "Fangiomics: revealing adaptive omics pharmacological mechanisms of the myriad combination therapies to achieve personalized medicine" in $2015^{[3]}$, uses rational drug combinations with higher efficacy but fewer adverse effects in a controlled array of designs by systematically integrating diverse omics data on genomic, proteomic, and metabolomic interactions, in contrast to traditional "omics" techniques focusing on a certain level of cell, tissue, or organ ${ }^{[2]}$. Based on the integration of multi-scale omic data and quantitative modeling of the relationships between complex diseases and combination therapy, Fangjiomics-based combination therapy presents its feasibility to achieving precision medicine, which would ensure that patients receive the right treatment at the right dose and at the right time, with maximum efficacy and minimum side effects. In this special issue, we continued to introduce its applications to the discovery of rational combination therapy and precision medicine.

The emerging pharmacophenomics studies the orchestrated multi-target pharmacology of combination therapy and provides a systematical paradigm for the pharmacological study of Fangji ${ }^{[20]}$. With well-defined molecular mechanisms of Zhenghou at the level of multi-omics through phenomewide association study (PheWAS) and a suite of new phenomics technologies and platforms, pharmacophenomics may be used to characterize the drug-response phenome of Fangji and to identify the corresponding multiple therapeutic targets according to the TCM theory of Jun-Chen-Zuo-Shi. Pharmacophenomic study of Fangji will also lay a theoretical foundation for the new science of precision medicine.

\section{References}

1 Day D, Siu LL. Approaches to modernize the combination drug development paradigm. Genome Med 2016; 8: 115.

2 Liu J, Wang Z. Diverse array-designed modes of combination therapies in Fangjiomics. Acta Pharmacol Sin 2015; 36: 680-8.

3 Duan DD, Wang Z, Zhang BL, Wang YY. Fangjiomics: revealing adaptive omics pharmacological mechanisms of the myriad combination therapies to achieve personalized medicine. Acta Pharmacol Sin 2015; 36: 651-3.

4 Zhang B, Wang X, Li S. An integrative platform of TCM network pharmacology and its application on a herbal formula, Qing-Luo-Yin. Evid Based Complement Alternat Med 2013; 2013: 456747.

5 Liu ZC, Wang ZL, Huang CY, Fu ZJ, Liu Y, Wei ZC, et al. Duhuo Jisheng Decoction inhibits SDF-1-induced inflammation and matrix degradation through the CXCR4/NF-kB pathway in human degenerative nucleus pulposus cells. Acta Pharmacol Sin 2018; 39: 912-22.

6 Luo YH, Zhu M, Wang DG, Yang YS, Tan T, Zhu H, et al. Yangxin Tongmai formula ameliorates impaired glucose tolerance in children with Graves' disease through upregulation of the insulin receptor levels. Acta Pharmacol Sin 2018; 39: 923-9.

7 Klayman DL. Qinghaosu (artemisinin): an antimalarial drug from China. Science 1985; 228: 1049-55.

8 McPhee DJ. Dr Kenneth A Jacobson: First winner of the Tu Youyou Award, in honor of the co-recipient of the 2015 Nobel Prize in physiology or medicine. Molecules 2016; 21. pii: E1656. 
9 Chen G, Xue WD, Zhu J. Full genetic analysis for genome-wide association study of Fangji: a powerful approach for effectively dissecting the molecular architecture of personalized traditional Chinese medicine. Acta Pharmacol Sin 2018; 39: 906-11.

10 Dong S, Cai FF, Chen QL, Song YN, Sun Y, Wei B, et al. Chinese herbal formula Fuzheng Huayu alleviates $\mathrm{CCl}_{4}$-induced liver fibrosis in rats: a transcriptomic and proteomic analysis. Acta Pharmacol Sin 2018; 39 : 930-41.

11 Song YN, Chen J, Cai FF, Lu YY, Chen QL, Zhang YY, et al. A metabolic mechanism analysis of Fuzheng-Huayu formula for improving liver cirrhosis with traditional Chinese medicine syndromes. Acta Pharmacol Sin 2018; 39: 942-51.

12 Zhang GX, Zhang YY, Zhang XX, Wang PQ, Liu J, Liu Q, et al. Different network pharmacology mechanisms of Danshen-based Fangjis in the treatment of stable angina. Acta Pharmacol Sin 2018; 39: 952-60.

13 Wang PQ, Liu Q, Xu WJ, Yu YN, Zhang YY, Li B, et al. Pure mechanistic analysis of additive neuroprotective effects between baicalin and jasminoidin in ischemic stroke mice. Acta Pharmacol Sin 2018; 39: 961-74.

14 Zhang YQ, Guo QY, Li QYI, Ren WQ, Tang SH, Wang SS, et al. Main active constituent identification in Guanxinjing capsule, a traditional
Chinese medicine, for the treatment of coronary heart disease complicated with depression. Acta Pharmacol Sin 2018; 39: 975-87.

15 Torkamani A, Andersen KG, Steinhubl SR, Topol EJ. High-definition medicine. Cell 2017; 170: 828-43.

16 Barabasi AL, Gulbahce N, Loscalzo J. Network medicine: a networkbased approach to human disease. Nat Rev Genet 2011; 12: 56-68.

17 Sun X, Vilar S, Tatonetti NP. High-throughput methods for combinatorial drug discovery. Sci Transl Med 2013; 5: 205rv1.

18 Zhang YQ, Wang C, Guo QY, Zhu CY, Yan C, Sun DN, et al. Molecular mechanisms of the analgesic action of Wu-tou Decoction on neuropathic pain in mice revealed using microarray and network analysis. Acta Pharmacol Sin 2018; 39: 988-97.

19 Wang FJ, Wang SX, Chai L, Zhang Y, Guo H, Hu LM. Xueshuantong injection (lyophilized) combined with salvianolate lyophilized injection protects against focal cerebral ischemia/reperfusion injury in rats through attenuation of oxidative stress. Acta Pharmacol Sin 2018; 39: 998-1011.

20 Duan DD, Han YS, Li L, Zhao JZ, Wang Z. Pharmacophenomics: a new paradigm for pharmacology, toxicology, and personalized medicine. Chin J Pharmacol Toxicol 2014; 28: 1-9. 